\title{
Experimental Investigation and Visual Observation of a Vapor-Liquid Separated Flat Loop Heat Pipe Evaporator
}

Yang Yang ${ }^{1}$, Zhu kai ${ }^{1}$, Wang Yabo ${ }^{1}$, Wei Jie ${ }^{2}$, Zhen Mingzhu ${ }^{1}$, Cui Zhuo ${ }^{1}$

(1. Tianjin University of Commerce, Key Laboratory of Refrigeration Technology of Tianjin, 300134, China)

(2. Fujitsu LTD. 2118588, Japan)

\begin{abstract}
It is known that the circulation driven head of heat pipe is mainly determined by both the vapor pressure head and the liquid pressure head generated at the phase change interface. In this paper, a unique operation mechanism is proposed; in this mechanism, the circulation of the loop heat pipe (LHP) is primarily driven by the phase change formed at the vapor-liquid interface. To test the new mechanism, a visual flat LHP evaporator prototype and an open experimental system were deliberately designed and assembled to obtain the operation characteristics inside of the evaporation chamber (EC). Three main experimental stages, the pre-boiling, boiling and steady stages, were observed during the start-up process. The evaporation chamber with $5-\mathrm{mm}$ height whose evaporator thermal resistance and LHP thermal resistance are $0.015{ }^{\circ} \mathrm{C} / \mathrm{W}$ and $0.36{ }^{\circ} \mathrm{C} / \mathrm{W}$, respectively, has a better heat transfer performance. The results showed that the circulation driven head formed inside of the EC played an important role in promoting the operation performance, especially when the wick, the vapor-liquid interface and the bottom of the evaporator arrived at a reasonable situation.
\end{abstract}

Key words: capillary loop heat pipe; flat heat pipe evaporator; wick; vapor-liquid interface; visualization

\section{Introduction}

As is known, the cooling device for high heat flux components is critical to promote the runtime reliability, optimize the performance and extend the lifetime of the product. With continuous development of large application servers, data processing centers and aerospace equipment, research and development of cost-effective thermal management become increasingly important to corresponding enterprises [1].

Typically, the waste heat output produced by a single Central Processing Unit (CPU) can achieve a total of more than $25-50 \mathrm{~W} / \mathrm{cm}^{2}$ and is expected to show a future rise with the increase in processor clock speed, the decrease of figure size and package space [2-5]. Therefore, the development speed and scale of large application servers and data centers will largely be affected by the research and development progress of its cooling devices.

The first flat shaped evaporator was patented by Yu. F. Gerasimov et al. in 1975 [6]. This evaporator aroused worldwide research interests in the thermal management and heat dissipation field, including structural optimization of LHP [7-8], heat and mass transfer process studies in the internal of LHP [9-10], wick selection and fabrication [11-14], working medium selection and charging principle [15], performance and reliability study [16-18], etc. Because LHP played an irreplaceable role in ensuring high heat flux components operate within a safe level [19], determining how to improve the LHP performance became the core field of study of many scholars [20-21]. In fact, the wick itself was the key factor to determine the capillary absorption rate in the heat transfer process of capillary wick loop heat pipe. Hence, most of the research revolves around the performance parameters, such as the thermal 
conductivity, porosity, permeability and capillary radius of the wick, charge ratio, heat load and backflow angle [22-23].

A loop heat pipe is a closed high efficiency thermal control device that utilizes capillary force to drive the working medium to complete the evaporation and condensing process in the internal of the system [24]. According to the purpose, working fluid, surface shape and dimensions of the heat source, either a flat or cylindrical evaporator is chosen for use. Because most surface shapes of the heat source are flat, flat evaporators have been more widely used than cylindrical evaporators. For a flat loop heat pipe, the flat evaporator was designed to be a sealed chamber; and a wick would be installed inside of the chamber. In addition, a condenser must be connected to the evaporator by two separate pipes to form a closed loop.

The structural setting of traditional LHP and CWHP will lead to a greater flow resistance, turning heat transfer efficiency limits into an unavoidable problem [25-26]. As a part of a traditional LHP and CWHP evaporator, the wick plays a vital function in driving liquid medium at condenser section flow back to evaporation section to maintain the operation process. The driving force mainly generated in the phase change interface of meniscus in the pore [27]. The main features and common problems of capillary phase change mechanism are: (1) two-phase flow will generate in the LHP due to the vapor-liquid interface existing in the wick during operation; (2) the parasitic heat leak in the evaporator increases its circulation flow resistance [28-30]. The inevitable problems above have extremely negative impacts on the further application.

In this paper, a flat loop heat pipe mainly driven by the phase change generated at the vapor-liquid interface inside of EC was proposed, and the working medium in the system could only perform one-way circulation, as shown in Fig. 1, i.e., reduced heat leak in the evaporator. The main heat transfer mechanism difference between this FLHP and the traditional FLHP was a phase change space was placed between the wick and the heating surface to remove the vapor-liquid interface from wick, which was called the evaporation chamber (EC). Because of the vapor-liquid interface was not inside of the wick any more, the new heat transfer mechanism utilized the vapor generated in the EC mentioned above to drive working medium to complete the circulation. The region between wick and the bottom of the evaporator prevents boiling phenomenon in the wick, so the shear flow resistance caused by the phase change and the partial pressure of vapor in the wick was near to zero. Thus, the flow resistance would then be reduced under the same seepage pressure in the wick. At the outlet of the vapor chamber, a buffer space, namely, the pressure sharing chamber, was designed to maintain the working medium in the vapor chamber realizing unidirectional phase of liquid to vapor to avoid the phase change phenomenon in the wick.

To ensure the boiling will not occur in the wick, the subcooling degree of the back flow and the height of the EC shall be kept at a reasonable value. In addition, a wick with lower thermal conductivity and suitable porosity is also required in the evaporator. Thus, the heat load applied to the bottom of the evaporator could only maintain the liquid evaporation in the evaporation chamber, and only a little heat could be transferred to the wick above. Therefore, the structure design of this paper could ensure that no reverse flow will occur in the wick and the flow resistance of vapor to the outlet was much less than the flow resistance of vapor to the wick. 
An excellent LHP is contributed by the good start-up and operational performance. The start-up performance usually can be evaluated with the start-up time, the minimum start-up heat load and the maximum operation heat load. In addition to the start-up performance experiment, the optimum height of EC was another major research topic of this paper. Based on the theory of heat transfer mechanism expressed above, this paper analyzes the start-up experiments and relevant thermal resistance under different evaporation chamber heights and their relations.

\section{Experiment apparatus and procedures}

\subsection{Experiment apparatus}

For the nontraditional capillary loop heat pipe system, the heat and mass transfer process in the EC had a decisive impact on the feasibility of above theory. As a core part of this type of LHP, an evaporator with visualization structure was designed to observe heat and mass transfer process, which had a vital impact on the thermal control performance of LHP.

The experiment system mainly consisted of : (1) a data acquisition system, (2) a DC regulated power supply, (3) water supply system, (4) an open-type visualization LHP evaporator, (5) a simulated CPU heat source, (6) high-speed camera (7) and a closed cooling tank, as depicted in Fig. 2. The evaporator assembly composed of a wick, an evaporation chamber (EC) below the wick, a pressure-sharing chamber structure at the outlet of the EC, a compensation chamber above the wick, vapor removal outlet and the liquid supply inlet. To obtain a directly observation and explore its operation mechanism inside of the evaporator during its operation process, the lateral shell of the evaporator was made of four transparent quartz glasses, each of thickness of $10 \mathrm{~mm}$. The lateral shells were bonded with heat-resistant adhesive and were successfully proven to withstand the external mechanical-shock and thermal-shock during the test. The top plate and the base plate of the evaporator were rectangular in shape with a planar dimension of $130 \mathrm{~mm}(\mathrm{~L}) \times 110 \mathrm{~mm}(\mathrm{~W})$, which were designed and machined with brass material, and the heating source connected with base plate was $75 \mathrm{~mm}(\mathrm{~L}) \times 50 \mathrm{~mm}(\mathrm{~W})$. Before the test, water in the supply system was degassed and cooled to the ambient temperature.

The molecular sieve with lower thermal conductivity was first used as the wick (porosity $40 \%, 20-\mathrm{mm}$ thickness) to reduce the heat conduction of the bottom plate to the wick and compensation chamber. A silicon heating plate of $25 \mathrm{~mm}(\mathrm{~L}) \times 25 \mathrm{~mm}(\mathrm{~W}) \times 2 \mathrm{~mm}(\mathrm{H})$ was placed at the bottom of one I-type copper block to simulate the CPU heating process and the heating power was adjusted by a DC power supply. In addition, a carbon fiber film with thermal conductivity of $75 \mathrm{~W} / \mathrm{m} \cdot \mathrm{K}$ was placed between the bottom plate of the evaporator and the copper block to reduce the thermal contact resistance, and the evaporator was bolted to the base with four bolts, as depicted in Fig. 3.

Yokogawa GP10 Data Acquisition System was applied to record temperature data with 
eighteen T-type (Copper-constantan Thermocouples) thermocouples. The layout of the detailed measuring points and the cross-section structure of the evaporator were depicted in Fig. 4 and Fig. 5, respectively.

In particular, three thermocouples were placed at the top plate for temperature measurement $\left(T_{1}-T_{3}\right)$. $T_{4}$ was used to measure the temperature above the wick. $T_{5}-T_{7}$ were placed at the upper wick to monitor the temperature changes. $\mathrm{T}_{6}$ was used for measuring the water supply temperature. $\mathrm{T}_{17}$ was used for measuring the water temperature in the water supply tank. $T_{9}$ was for monitoring the vapor temperature at the outlet of the evaporator. $T_{10^{-}}$ $T_{12}$ were inserted into the bottom plate of the evaporator to measure the wall temperature. $T_{13^{-}}$ $\mathrm{T}_{16}$ were inserted into the copper block to calculate the actual heating power. Another thermocouple was used to measure ambient temperature. $T_{1}-T_{12}, T_{17}-T_{18}(0.2-m m$ diameter $)$ and $\mathrm{T}_{13}-\mathrm{T}_{16}(0.1-\mathrm{mm}$ diameter $)$ had an accuracy of $\pm 0.2{ }^{\circ} \mathrm{C}$.

\subsection{Test procedures}

Before the test, distilled water was charged into the liquid tank to prevent the experiments from being affected by impurities [31]. During the test, all evaporator experiment system components were horizontally placed at the test bench, except for the liquid tank and the vapor cooling tank. The liquid tank was placed above the evaporator, and the vapor cooling tank was placed below the evaporator. Furthermore, all of the temperature readings were recorded using a data acquisition system, and the flow states in the evaporator were captured via a high-speed camera.

This paper mainly focuses on the heat and mass transfer process inside of the evaporator, so an open experimental system was deliberately designed. Therefore, observation of stable vapor generated inside of the EC during the start-up process shall be the complete start-up judgment criterion. The evaporator start-up test was conducted under different EC heights of $1 \mathrm{~mm}$ and $5 \mathrm{~mm}$. Before the test beginning, $1 \mathrm{~mm}$ of water was charged into the EC to simulate the actual distribution of water under the influence of gravity.

To observe stable state operation characteristics inside of the evaporator after each heating power adjusting process, the time of the test curves would be longer during the tests.

\section{Results and discussion}

\subsection{Evaporator start-up experiment}

According to the temperature changes of the evaporator outlet, the whole start-up process could be divided into three stages: pre-boiling stage (before $T_{9}$ changing), boiling stage (during the changing of $T_{9}$ ) and stabilization boiling stage ( $T_{9}$ stop changing). Fig. 6 and Fig. 7 show the temperature change of evaporator start-up process under the condition of 1 $\mathrm{mm}$ and 5-mm EC heights. The effective heating power applied to the base plate was $113.5 \mathrm{~W}$ $\left(3.75 \mathrm{~W} / \mathrm{cm}^{2}\right)$. The start-up time of the evaporator refers to the time from the start of heating to the stable operation. The main operation parameters under different EC heights are listed in 
Table 2. For the 1-mm EC height condition, the start-up time is $800 \mathrm{~s}$, and the boiling phenomenon starts at $330 \mathrm{~s}$. For the 5-mm EC height condition, the start-up time is $750 \mathrm{~s}$, and the boiling phenomenon starts at $130 \mathrm{~s}$. Fig. 6 shows that the temperature curves were no longer changed at $800 \mathrm{~s}$ when steady state was considered to be reached. Fig. 7 shows that the temperature curves were no longer changed at $750 \mathrm{~s}$, when steady state was considered to be reached.

Fig. 8 visually shows the operation status inside both of the wick and the EC after the water dropped from wick to the evaporator base plate at the stabilization boiling stage under the 5-mm EC height condition, and the effective heating power was $113.5 \mathrm{~W}\left(3.75 \mathrm{~W} / \mathrm{cm}^{2}\right)$. In addition, the drawings in the right of the picture were made to more directly and clearly display of the mass transfer process inside of the evaporator.

Based on the research of the start-up process in this paper, the liquid at the initial level touched the lower surface of the wick under the 1-mm EC height condition. Before complete start-up, the boiling phenomenon appeared slowly because part of the heat applied to the bottom plate could be transferred to the wick through the liquid in the EC, reducing the heat applied to the liquid itself. For the 5-mm EC height condition, however, more heat would be applied to the liquid because of the wick did not directly touch to the liquid, which shortening the boiling time in the EC.

The heat applied to water almost had no heat leak to the wick in pre-boiling stage, when EC height was raised up to $5 \mathrm{~mm}$. That situation changed as the heating went on; the stable vapor bubble appeared and was broken up inside of the EC, making it possible for the heat transferred from the bottom plate to the wick above. At the same time, the heat radiation between EC and the wick caused the wick temperature to rise faster. The stabilization boiling stage appeared when wall superheat reached and remained at $2.1^{\circ} \mathrm{C}$. The wall temperature and vapor temperature no longer increased with the growing heat load. Early in the boiling stage, part of the vapor in the pressure-sharing chamber condensates into dew and then flow back to the EC or remains at the vapor line. Therefore, the growth of $\mathrm{T}_{9}$ lagged behind the growth of $\mathrm{T}_{10}-\mathrm{T}_{12}$, as shown in Fig. 6 and Fig. 7. As the vapor generated in the EC constantly was discharged from the evaporator, the liquid tank should provide water supply through adjusting the liquid line valve. Thus, a complete cycle of the open-loop experiment system was completed.

\subsection{Variable heat load experiment}

Fig. 9 shows the profile of temperature change during variable heat load experiment under the 1-mm EC height condition, and some of main operation parameters are listed in Table 3. During the test, the initial heat load of the 1-mm EC condition was $10 \mathrm{~W}$, and the 
heat load was gradually increased by $10 \mathrm{~W}$.

According to the vapor temperature change profile in Fig. 9, the experiment process could also be divided into three stages: low heat load stage, high efficient cycle stage and dryout stage. Under the 1-mm EC height condition, the evaporator worked in low heat load stage when the heat load is less than $70 \mathrm{~W}\left(2.31 \mathrm{~W} / \mathrm{cm}^{2}\right)$. Because the cycle had not been established, the heat applied to the base plate could not be efficiently removed, resulting in the temperature rise of the evaporator. Although some vapor bubbles appeared in the EC during this stage of the heat load continually rising, it actually belonged to "subcooled boiling". The vapor would re-condensate inside of the EC and flow back to the bottom plate, resulting in the drastic vapor temperature fluctuation phenomenon at the evaporator outlet in Fig. 9. When the heat load was greater than $70 \mathrm{~W}$, the bottom plate temperature would further increase, and then the superheat degree between bottom plate temperature and the saturation temperature of water corresponding to the atmosphere pressure would also further increase. Afterwards, stabilization boiling formed, and the heat applied to the bottom plate could be taken away through vapor-liquid phase change occurring inside of the EC, and then the heat was transferred to the cooling tank in the end. At this time, the bottom plate temperature tended to be stable, despite the increase of heat load, and the bottom plate temperature was finally under effective control.

Fig. 10 shows the profile of temperature change during variable heat load experiment under the 5-mm EC condition; and some of the main operation parameters are listed in Table 3. To reduce the experimental time, the initial heat load condition was $20 \mathrm{~W}$, and the heat load was gradually increased by $20 \mathrm{~W}$ until a dry-out phenomenon appeared inside of the $5-\mathrm{mm}$ EC.

Boiling phenomenon in the EC was difficult to achieve when heat load less than $60 \mathrm{~W}$ $\left(1.98 \mathrm{~W} / \mathrm{cm}^{2}\right)$ because the evaporator was in the process of heat storage and the superheat degree between the bottom plate temperature and the saturation temperature of water corresponding to the atmosphere pressure could not reach to the required value. As the heating power continually increased and exceeded $80 \mathrm{~W}$, bubbles began to generate in the EC and the T9 started to fluctuate heavily. The circulation was successfully set up when stable vapor generated and leaved from the EC after heat power reached to $100 \mathrm{~W}$. Film-shaped boiling was maintained when heat power reached to $120 \mathrm{~W}$. The three temperature points in the bottom plate only rose slightly and other temperature points were also in steady state, such as the thermocouples above the wick and the water supply pipe. However, a sharp rise of temperature in the bottom plate was observed when heating power reached to $220 \mathrm{~W}$ (7.26 $\mathrm{W} / \mathrm{cm}^{2}$ ), a large amount of vapor remained inside of the EC and made it difficult for the water in the wick to drop. Finally, a phenomenon of complete drying was observed in the EC. Therefore, we conclude from the above information that there was a relationship between the heating power and the EC height.

Some meaningful results could be obtained through the experiments above: the reason for the sharp temperature rise generated in the bottom plate was that the heat absorbed by the 
wick was far less than the heat applied to the bottom plate, leading to the heat remained at the bottom. No dry-out phenomenon was observed in the EC within the heating range of the test when EC height was $1 \mathrm{~mm}$, but there was a heating limit when the EC was $5 \mathrm{~mm}$. A higher EC height was beneficial to reduce the thermal resistance, and a smaller EC height could help to prevent the dried up phenomenon from occurring in the evaporator during the test.

\subsection{Thermal resistance}

Generally, evaporator thermal resistance and total thermal resistance are important parameters that reflect the performance of LHP during the operation [16, 32-33]; those two thermal resistances are defined as:

$$
\begin{aligned}
& \mathrm{R}_{\mathrm{e}}=\frac{\mathrm{T}_{\mathrm{e}}-\mathrm{T}_{\mathrm{v}}}{\mathrm{Q}}(1) \\
& \mathrm{R}_{\text {total }}=\frac{\mathrm{T}_{\mathrm{j}}-\mathrm{T}_{\mathrm{a}}}{\mathrm{Q}}(2)
\end{aligned}
$$

where $\mathrm{Q}$ is the heat load applied to the base plate, and Q is obtained by calculating the temperature monitored in the copper block; $\mathrm{T}_{\mathrm{e}} \mathrm{wa}$ is the average temperature of $\mathrm{T}_{10}-\mathrm{T}_{12} ; \mathrm{T}_{\mathrm{v}}$ wais the vapor temperature at the evaporator outlet $\left(T_{9}\right)$; $T_{j}$ wais the actual temperature in the middle of simulated CPU surface $\left(\mathrm{T}_{12}\right) ; \mathrm{T}_{\mathrm{a}}$ wais the ambient temperature.

Fig. 11 to Fig. 14 show the evaporator resistance and the total thermal resistance change profile of the cases of 1-mm EC height and 5-mm EC height, respectively. As the experiment results showed, the minimum evaporator thermal resistance and total thermal resistance of the 1-mm EC height condition was $0.065{ }^{\circ} \mathrm{C} / \mathrm{W}$ and $0.4{ }^{\circ} \mathrm{C} / \mathrm{W}$, respectively. In addition, the minimum evaporator thermal resistance and total thermal resistance of the 5-mm EC height condition were $0.015{ }^{\circ} \mathrm{C} / \mathrm{W}$ and $0.36{ }^{\circ} \mathrm{C} / \mathrm{W}$, respectively.

The results showed that both the evaporator thermal resistance and the total thermal resistance of the 5-mm EC height condition were less than that of the 1-mm EC height condition. This behavior occurred because the boiling efficiency and vapor discharge efficiency e improved with increasing EC height. In addition, the evaporator thermal resistance and total thermal resistance had the similar changing trend with the increase of heat load. Before steady circulation was achieved, the whole evaporator functioned as a heat conductor, and it was cooled only by natural convection and radiation with surroundings, resulting in a high evaporator thermal resistance and total thermal resistance. Once the steady circulation was achieved, the evaporator thermal resistance and total thermal resistance could be reduced to a lower level, and the thermal resistance decreased with the increasing EC height. Thus, it was better to evaluate this type of LHP with total thermal resistance under the current condition.

\section{Conclusions}

In this paper, a visualization flat loop heat pipe evaporator with a special structure was 
described, for which the heating surface had no direct contact with the wick, i.e., the vaporliquid interface was separated from the wick. This type of LHP was mainly driven by the phase change generated inside of the EC to complete the single direction circulation. This paper mainly focused on the relationship between the EC height and the vapor-liquid interface location, as well as the influence of EC height change to the boiling efficiency during start-up and variable heat load experiments. Based on the experimental results, the following conclusions could be drawn:

1. According to the vapor temperature at the evaporator outlet under heat load of 113.5 $\mathrm{W}$, the start-up process can be divided into three stages: a pre-boiling stage, boiling stage and stabilization boiling stage. The start-up time of the 5-mm EC height condition is less than that of the 1-mm EC height condition.

2. The variable heat load experiment process can also be divided into three stages: low heat load stage, high efficient cycle stage and dry-out stage. A dry-out phenomenon appears in the 5 -mm height condition when heat load is increased to $220 \mathrm{~W}$ and the vapor-liquid interface appears in the wick. However, the same phenomenon does not appear in the 1-mm height condition, i.e., a smaller EC is beneficial to restrain the generation of dry-out.

3. The minimum evaporator thermal resistance and the LHP total thermal resistance of the $1-\mathrm{mm}$ EC height condition are $0.065{ }^{\circ} \mathrm{C} / \mathrm{W}$ and $0.4{ }^{\circ} \mathrm{C} / \mathrm{W}$, respectively. In addition, the minimum evaporator thermal resistance and the LHP total thermal resistance of the 5-mm EC height condition are $0.015{ }^{\circ} \mathrm{C} / \mathrm{W}$ and $0.36{ }^{\circ} \mathrm{C} / \mathrm{W}$, respectively. A higher EC height is helpful to improve boiling efficiency, causing a sharp decrease of the evaporator thermal resistance and the total thermal resistance of LHP.

4. The new operation mechanism that the working fluid in LHP is mainly driven by the phase change generated inside of the EC is valid. Increasing the seepage force in the wick and maintaining a sufficient driven force in evaporator are the keys. The required conditions are ensuring the condensate has a certain degree of supercooling and finding an optimum height of the EC.

\section{Acknowledgments}

This work is supported by Project (No. 51376137) of the Chinese National Science Foundation and Project (No. 13JCZDJC27300) of the Chinese Tianjin City Science Foundation.

\section{NOMENCLATURE}

A area, $\mathrm{m}^{2}$

$\mathrm{T}$ temperature, ${ }^{\circ} \mathrm{C}$

$\mathrm{R}$ thermal resistance, ${ }^{\circ} \mathrm{C} / \mathrm{W}$ 


\section{References}

[1] Singh R, Akbarzadeh A, Mochizuki M. Sintered porous heat sink for cooling of highpowered microprocessors for server applications [J]. International Journal of Heat and Mass Transfer, 2009, 52(9): 2289-2299.

[2] Maydanik Y F, Vershinin S V, et al. Miniature Loop Heat Pipes - a Promising Means for Cooling Electronics. IEEE Transactions on Components and Packaging Technologies, 2005, 28(2): pp.290-296

[3] Randeep S, Aliakbar A et al. Novel Design of a Miniature Loop Heat Pipe Evaporator for Electronic Cooling. Journal of Heat Transfer, 2007, 129(10): pp.1445-1452

[4] Vasiliev, L, Lossouarn, D., Romestant, C., et al, Loop heat pipe for cooling of high-power electronic components, International Journal of Heat and Mass Transfer (2008), Volume 52, Issues 1-2, 15 January 2009, pp.301-308

[5] Hoang TT, Ku J. Advanced loop heat pipes for spacecraft thermal control[C]/8th AIAA/ASME Joint Thermophysics and Heat Transfer Conference. St Louis, Missouri: AIAA, 2002 (AIAA-2002-3094):8-16

[6]Yu.F. Gerasimov, Yu.F. Maydanik, V.M. Kiseev, G.A. Philippov, L.G. Starikov. Evaporation Chamber of a Heat Pipe, USSR Inventor Certificate 495522, 1975.

[7]Kiseev V M, Vlassov V V, Muraoka I. Experimental optimization of capillary structures for loop heat pipes and heat switches [J]. Applied Thermal Engineering, 2010, 30(11): 1312-1319.

[8]CrepinsekM, ParkC. Experimental analysis of pump-assisted and capillary-driven dualevaporators two-phase cooling loop [J].Applied Thermal Engineering, 2012, 38: 133-142.

[9]Ren C, Wu Q S, Hu M B. Heat transfer with flow and evaporation in loop heat pipe's wick at low or moderate heat fluxes [J]. International Journal of Heat and Mass Transfer, 2007, 50(11): 2296-2308.

[10]Siedel B, Sartre V, Lefèvre F. Numerical investigation of the thermohydraulic behavior of a complete loop heat pipe [J].Applied Thermal Engineering, 2013, 61(2): 541-553.

[11]Yeh C C, Liu B H, Chen Y M. A study of loop heat pipe with biporous wicks [J]. Heat and Mass Transfer, 2008, 44(12): 1537-1547.

[12]Boo J H, Chung W B. Experimental study on the thermal performance of a small-scale loop heat pipe with polypropylene wick [J]. Journal of mechanical science and technology, 2005, 19(4): 1052-1061.

[13]Liu Z, Gai D, Li H, et al. Investigation of impact of different working fluids on the operational characteristics of miniature LHP with flat evaporator[J]. Applied Thermal Engineering, 2011, 31(16): 3387-3392.

[14]Joung W, Yu T, Lee J. Experimental study on the loop heat pipe with a planar bifacial wick structure [J]. International Journal of Heat and Mass Transfer, 2008, 51(7): 1573-1581.

[15]V.M. Kiseev, A.S. Nepomnyashy, N.L. Gruzdova, K.S. Kim, Miniature loop heat pipes for CPU cooling, in: Proceedings of the 7th International Heat Pipe Symposium, Jeju, Korea, 2003, pp. 175e180. 
[16] Singh R, Akbarzadeh A, Mochizuki M. Operational characteristics of a miniature loop heat pipe with flat evaporator [J]. International Journal of Thermal Sciences, 2008, 47(11): 1504-1515.

[17]Li J, Wang D, Peterson G P. Experimental studies on a high performance compact loop heat pipe with a square flat evaporator [J]. Applied Thermal Engineering, 2010, 30(6): 741752.

[18]Wang S, Zhang W, Zhang X, et al. Study on start-up characteristics of loop heat pipe under low-power [J]. International Journal of Heat and Mass Transfer, 2011, 54(4): 1002 1007.

[19] Deeney, J., "Thermal Modeling and Measurement of High Power Silicon Devices with Asymmetric Power Distribution," Proc of 35th IMAPS International Symposium on Microelectronics, 2002

[20] Prasher R. A Simplified Conduction Based Modeling Scheme for Design Sensitivity Study of Thermal Solution Utilizing Heat Pipe and Vapor Chamber Technology [J]. Electronic Packaging, Vol. 125, pp. 378-385, 2003

[21] Xu, G., Follmer, L. and Cooley J., Thermal solution development for the Sun Fire TM E25K server, Proceedings of SEMI-THERM21 (2005), San Jose, USA

[22] Randeep Singh, Thang Nguyen, Masataka Mochizuki. Capillary evaporator development and qualification for loop heat pipes [J]. Applied Thermal Engineering. 2014. 63 ( 1 ) : 406418

[23]S. Becker,S. Vershinin,V. Sartreet al. Steady state operation of a copper-water LHP with a flat-oval evaporator[J]. Applied Thermal Engineering. 2011. 31 ( 5 ) : 686-695

[24] Maydanik Y F, Chernysheva M A, Pastukhov V G. Review: Loop heat pipes with flat evaporators [J].Applied Thermal Engineering, 2014, 67(1): 294-307.

[25] Jentung Ku, Donya M. Douglas, Michael Pauken, Miniature Loop Heat Pipe with Multiple Evaporators for Thermal Control of Small Spacecraft, N.G.S.F. Center, Editor. 2004

[26] Hosei Nagano, Jentung $\mathrm{Ku}$, Capillary limit of a multiple-evaporator and multiplecondenser miniature loop heat pipe [J]. Journal of Thermophysics and Heat Transfer, 2007. 21(4), pp. 694

[27] Yu.F. Gerasimov, Yu.F. Maydanik, G.T. Shchogolev, etal. Low-temperature heat pipes with separate channels for vapor and liquid, Eng.-Phys. J. 28 (6) (1975) 957-960 (in Russian).

[28] Singh R, Nguyen T, Mochizuki M. Capillary evaporator development and qualification for loop heat pipes [J]. Applied Thermal Engineering, 2014, 63(1): 406-418.

[29] X.H. Nguyen, B.H. Sung, J. Choi, S.R. Ryoo, H.S. Ko, C. Kim, Study on heat transfer performance for loop heat pipe with circular flat evaporator, Int. J. Heat Mass Transfer 55 (4) (2012) $1304 \mathrm{e} 1315$.

[30] Xu J, Zhang L, Xu H. Performance of LHPs with a novel design evaporator [J]. International Journal of Heat and Mass Transfer, 2012, 55(23): 7005-7014. 
[31] JiayinXu, Li Zhang, Hong Xu, JieZhong, Jin Xuan. Experimental investigation and visual observation of loop heat pipes with two-layer composite wicks [J]. International Journal of Heat and Mass Transfer, 2014, 72: 378-387

[32] Singh R, Akbarzadeh A, Mochizuki M. Operational characteristics of a miniature loop heat pipe with flat evaporator [J]. International Journal of Thermal Sciences, 2008, 47(11): 1504-1515.

[33] Singh R, Akbarzadeh A, Dixon C, et al. Novel design of a miniature loop heat pipe evaporator for electronic cooling [J]. Journal of Heat Transfer, 2007, 129(10): 1445-1452. 
Table 1 Correspondence between the thermocouple code and thermocouple location

\begin{tabular}{cc}
\hline Code number & Thermocouple location \\
\hline $\mathrm{T}_{1}$ & Right of the top plate \\
$\mathrm{T}_{2}$ & Middle of the top plate \\
$\mathrm{T}_{3}$ & Left of the top plate \\
$\mathrm{T}_{4}$ & Upper side of the splitter plate \\
$\mathrm{T}_{5}$ & Upper middle of the wick \\
$\mathrm{T}_{6}$ & Fluid supply temperature in the compensation \\
$\mathrm{T}_{7}$ & chamber \\
$\mathrm{T}_{8}$ & Upper right of the wick \\
$\mathrm{T}_{9}$ & Evaporator outlet \\
$\mathrm{T}_{10}$ & Right of the base plate \\
$\mathrm{T}_{11}$ & Left of the base plate \\
$\mathrm{T}_{12}$ & Middle of the base plate \\
$\mathrm{T}_{13}$ & Copper block (first) \\
$\mathrm{T}_{14}$ & Simulated CPU \\
$\mathrm{T}_{15}$ & Copper block (second) \\
$\mathrm{T}_{16}$ & Copper block (third) \\
$\mathrm{T}_{17}$ & Fluid supply tank \\
$\mathrm{T}_{18}$ & Ambient \\
\hline &
\end{tabular}


Table 2 Key operation parameters of the different EC heights condition

\begin{tabular}{ccccc}
\hline $\begin{array}{c}\text { Height } \\
(\mathrm{mm})\end{array}$ & Boiling formation time & Superheat degree & Maximum wall temperature & \multicolumn{2}{c}{ Operation temperature } & $\left({ }^{\circ} \mathrm{C}\right)$ \\
\hline 1 & $(\mathrm{~s})$ & $\left({ }^{\circ} \mathrm{C}\right)$ & 108.7 & 107.6 \\
\hline 5 & 330 & 2.1 & 108.8 & 107.9 \\
\hline
\end{tabular}


Table 3 Variable heat load operation parameters of different EC heights

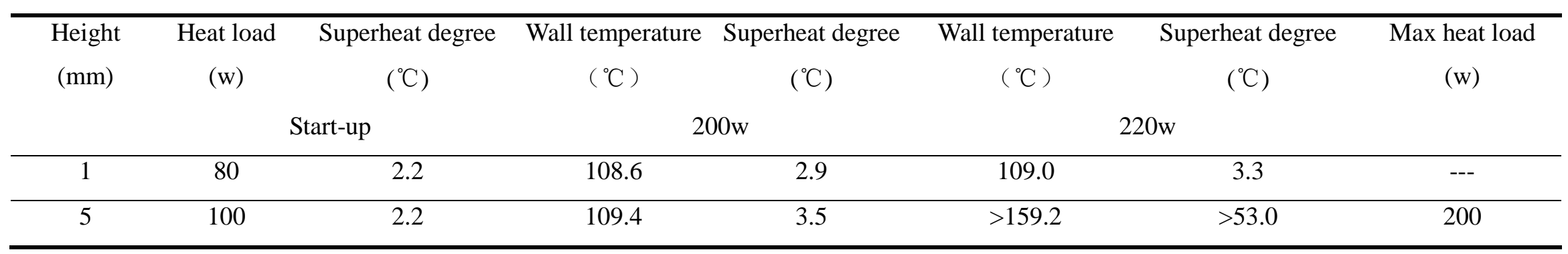


Table 2 Key operation parameters of different vapor chamber heights condition

\begin{tabular}{|c|c|c|c|c|c|}
\hline $\begin{array}{l}\text { Height } \\
(\mathrm{mm})\end{array}$ & $\begin{array}{c}\text { Boiling } \\
\text { formation time } \\
\text { (s) }\end{array}$ & $\begin{array}{l}\text { Superheat } \\
\text { degree } \\
\left({ }^{\circ} \mathrm{C}\right)\end{array}$ & $\begin{array}{c}\text { Maximum } \\
\text { wall } \\
\text { temperature } \\
\left({ }^{\circ} \mathrm{C}\right)\end{array}$ & $\begin{array}{l}\text { Operation } \\
\text { temperature } \\
\left({ }^{\circ} \mathrm{C}\right)\end{array}$ & $\begin{array}{l}\text { Start-up } \\
\text { time } \\
\text { (s) }\end{array}$ \\
\hline 1 & 330 & 2.1 & 108.7 & 107.6 & 800 \\
\hline 5 & 130 & 2.1 & 108.8 & 107.9 & 750 \\
\hline
\end{tabular}


Table 3 Variable heat load operation parameters of different vapor chamber heights

\begin{tabular}{|c|c|c|c|c|c|c|c|}
\hline$(\mathrm{mm})$ & $\begin{array}{l}\text { Heat } \\
\text { load } \\
\text { (w) }\end{array}$ & $\begin{array}{c}\text { Superheat } \\
\text { degree } \\
\left({ }^{\circ} \mathrm{C}\right)\end{array}$ & $\begin{array}{c}\text { Wall } \\
\text { temperature } \\
\left({ }^{\circ} \mathrm{C}\right)\end{array}$ & $\begin{array}{c}\text { Superheat } \\
\text { degree } \\
\left({ }^{\circ} \mathrm{C}\right)\end{array}$ & $\begin{array}{c}\text { Wall } \\
\text { temperature } \\
\left({ }^{\circ} \mathrm{C}\right)\end{array}$ & $\begin{array}{c}\text { Superheat } \\
\text { degree } \\
\left({ }^{\circ} \mathrm{C}\right)\end{array}$ & $\begin{array}{c}\text { Max heat } \\
\text { load } \\
\text { (w) }\end{array}$ \\
\hline & \multicolumn{2}{|c|}{ Start-up } & \multicolumn{2}{|c|}{$200 \mathrm{w}$} & \multicolumn{3}{|c|}{$220 \mathrm{w}$} \\
\hline 1 & 80 & 2.2 & 108.6 & 2.9 & 109.0 & 3.3 & --- \\
\hline 5 & 100 & 2.2 & 109.4 & 3.5 & $>159.2$ & $>53.0$ & 200 \\
\hline
\end{tabular}


Fig.1 The heat pipe driven by phase change force

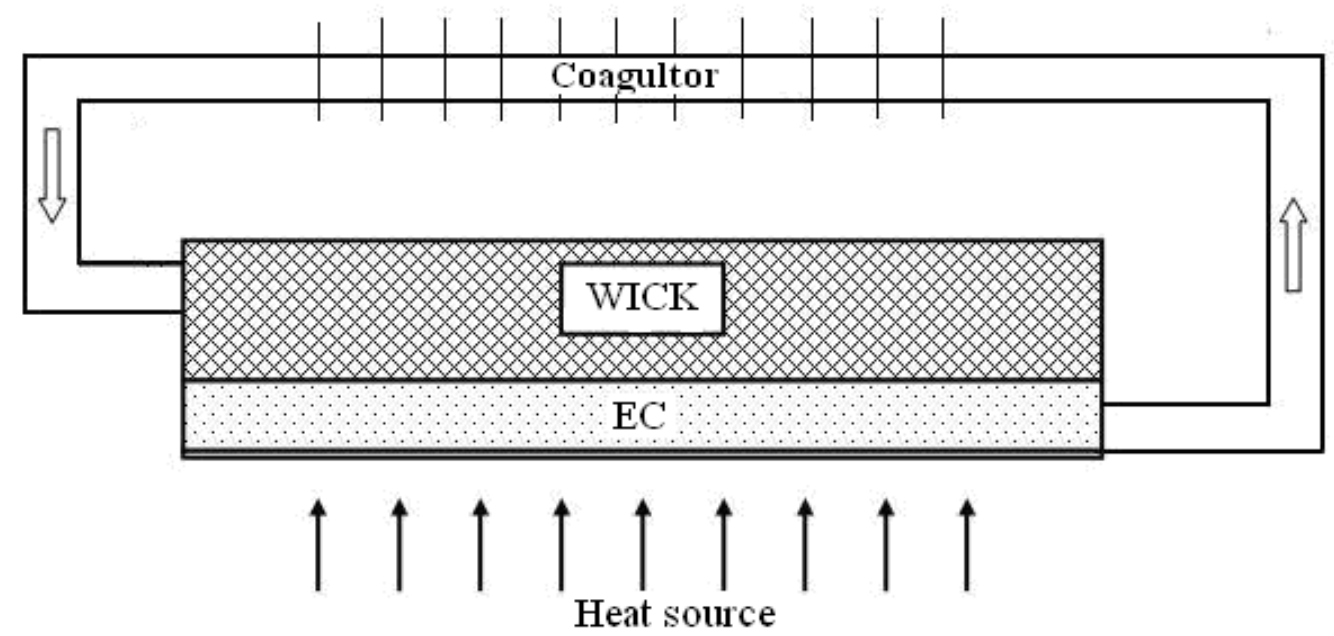


Fig.2 Schematic of experimental prototype evaporator




Fig.3 Schematic of the visualization LHP

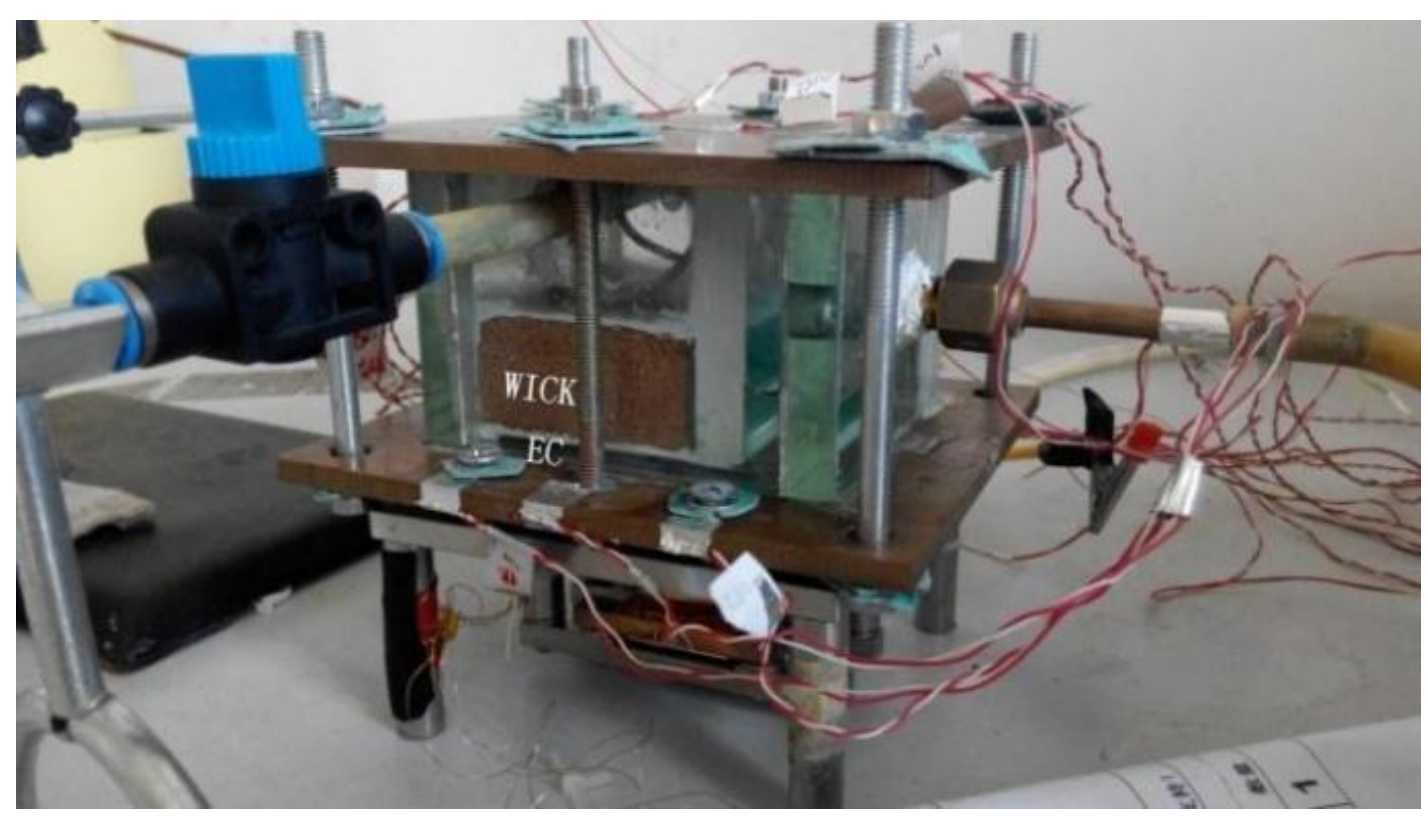


Fig.4 Test layout of the evaporator

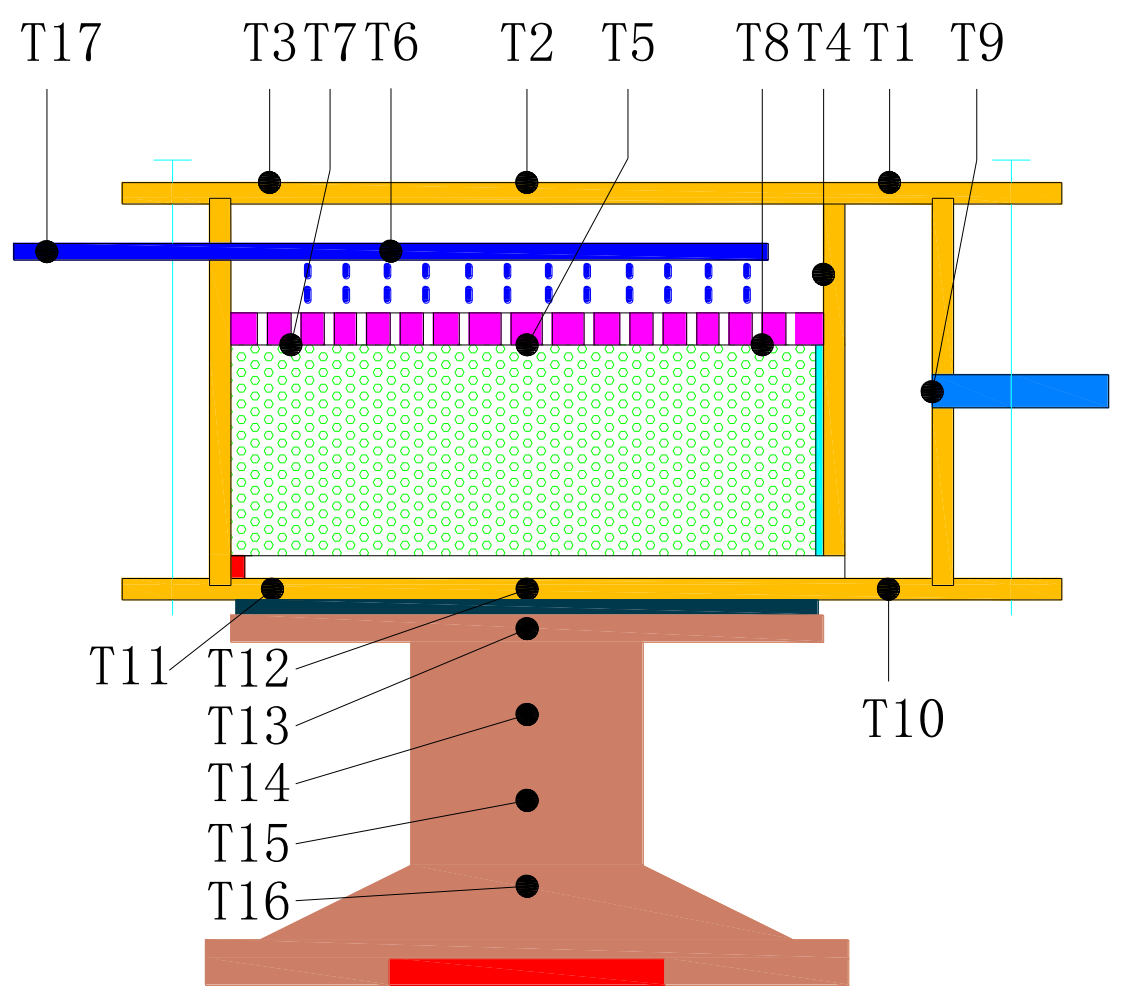


Fig.5 Cross-sectional details of the evaporator

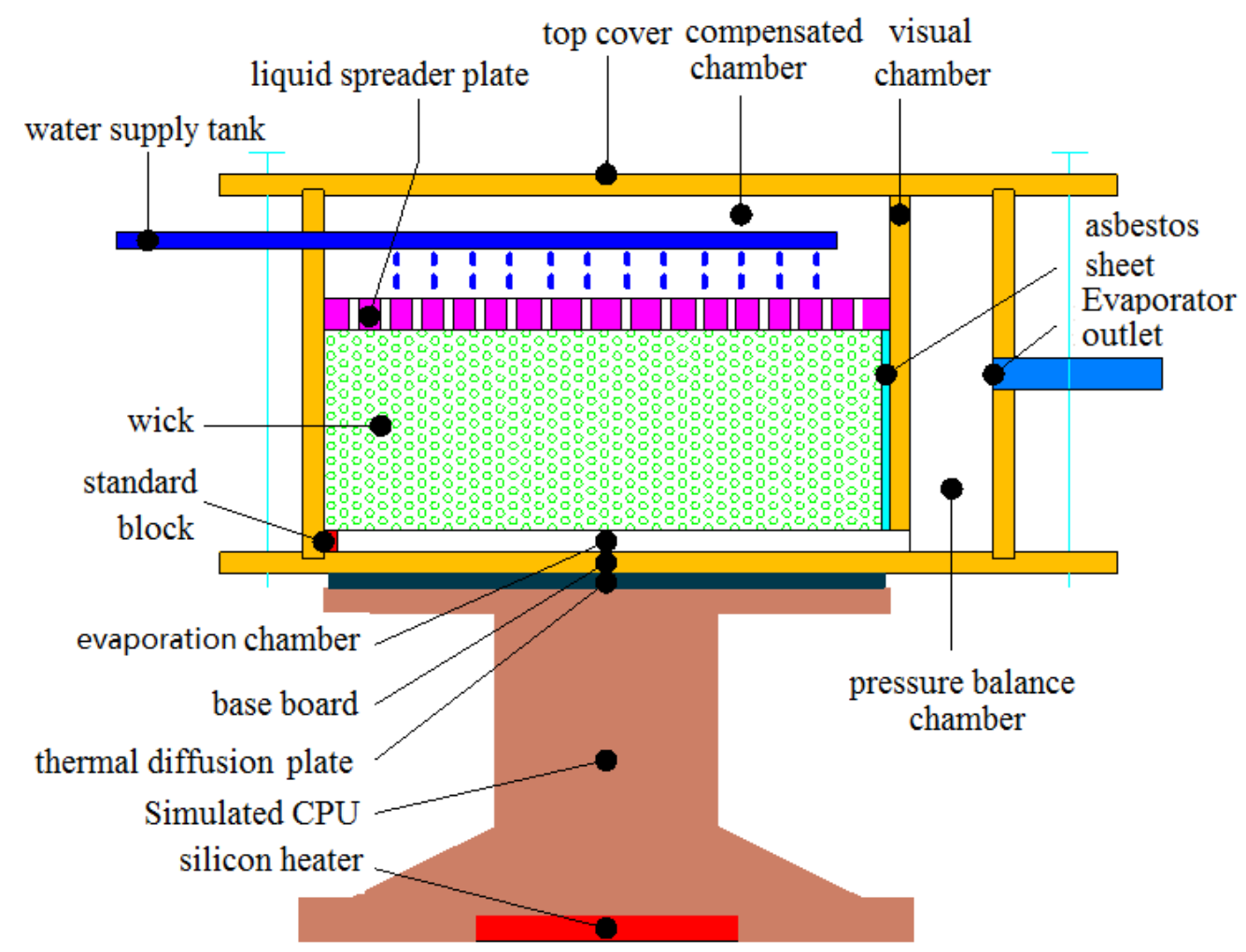


Fig.6 Temperature curve of the 1-mm EC during the start-up process

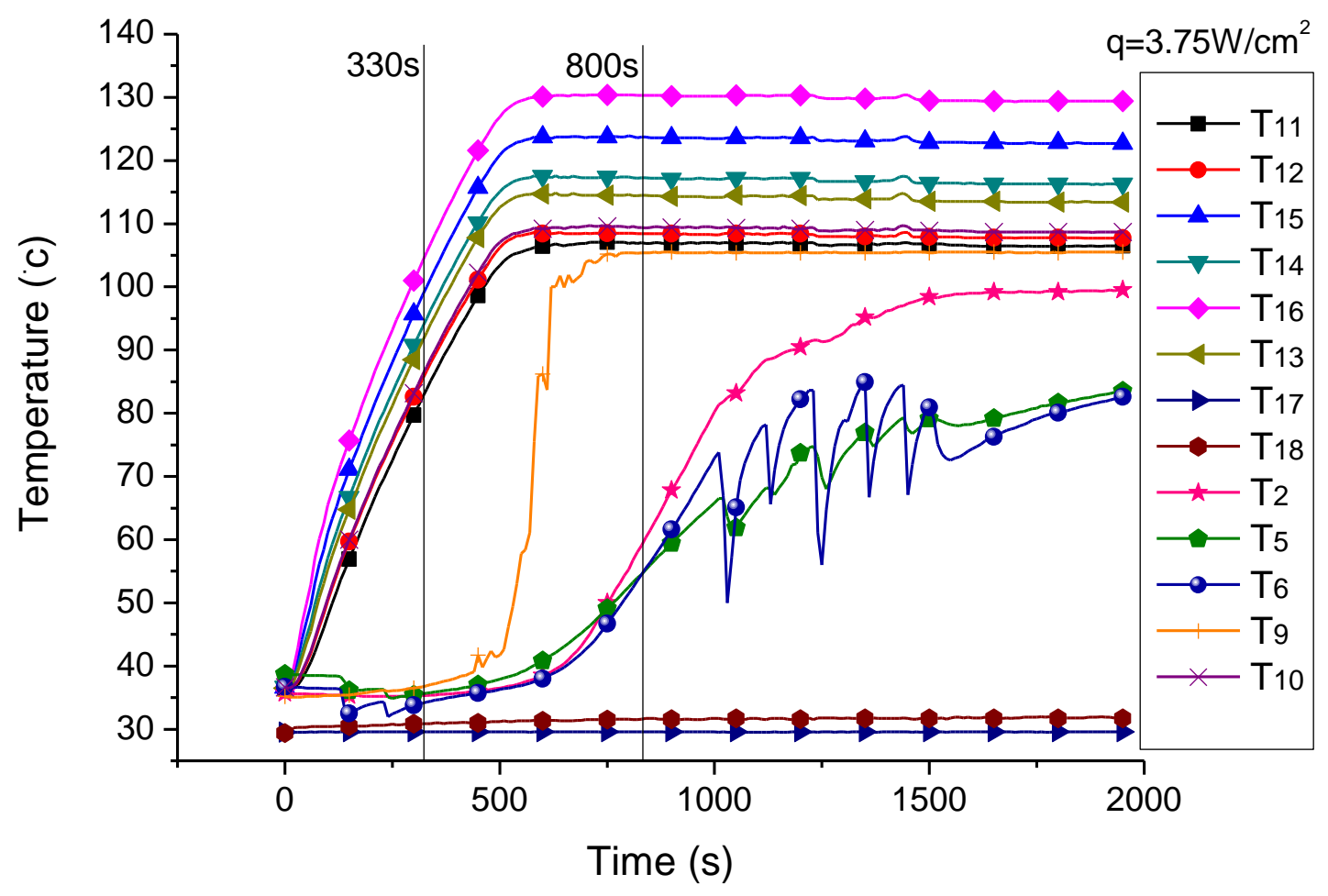


Fig.7 Temperature curve of the 5-mm EC during the start-up process

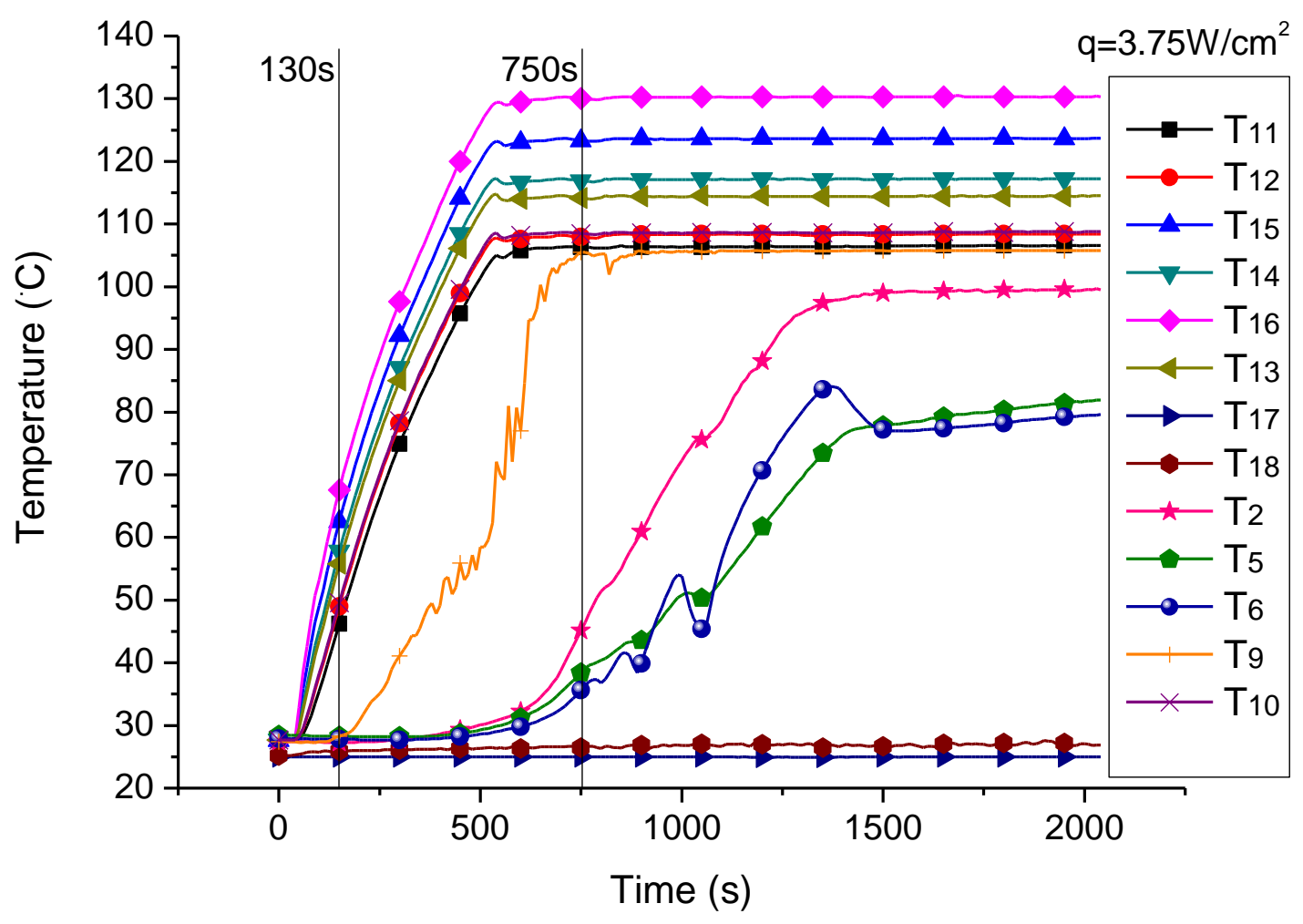


Fig.8 Stable evaporation process for the 5-mm EC during the start-up process
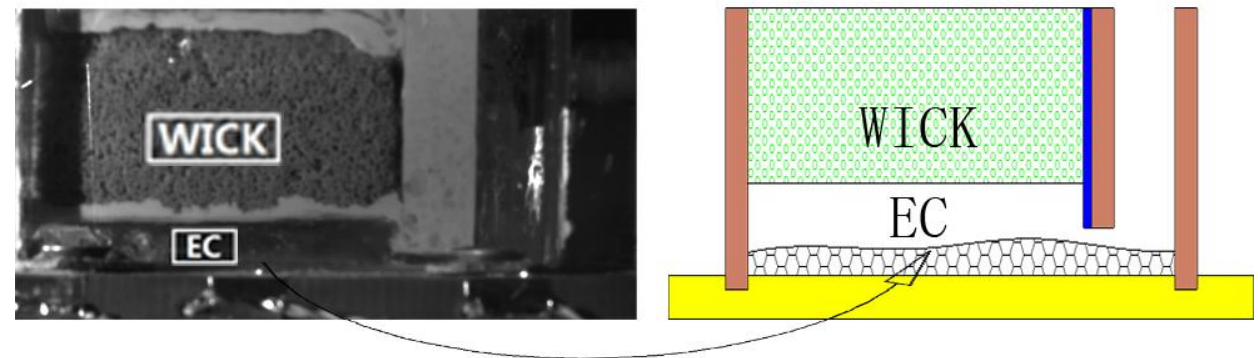
Fig.9 Variable heat load operation process of the 1-mm EC condition

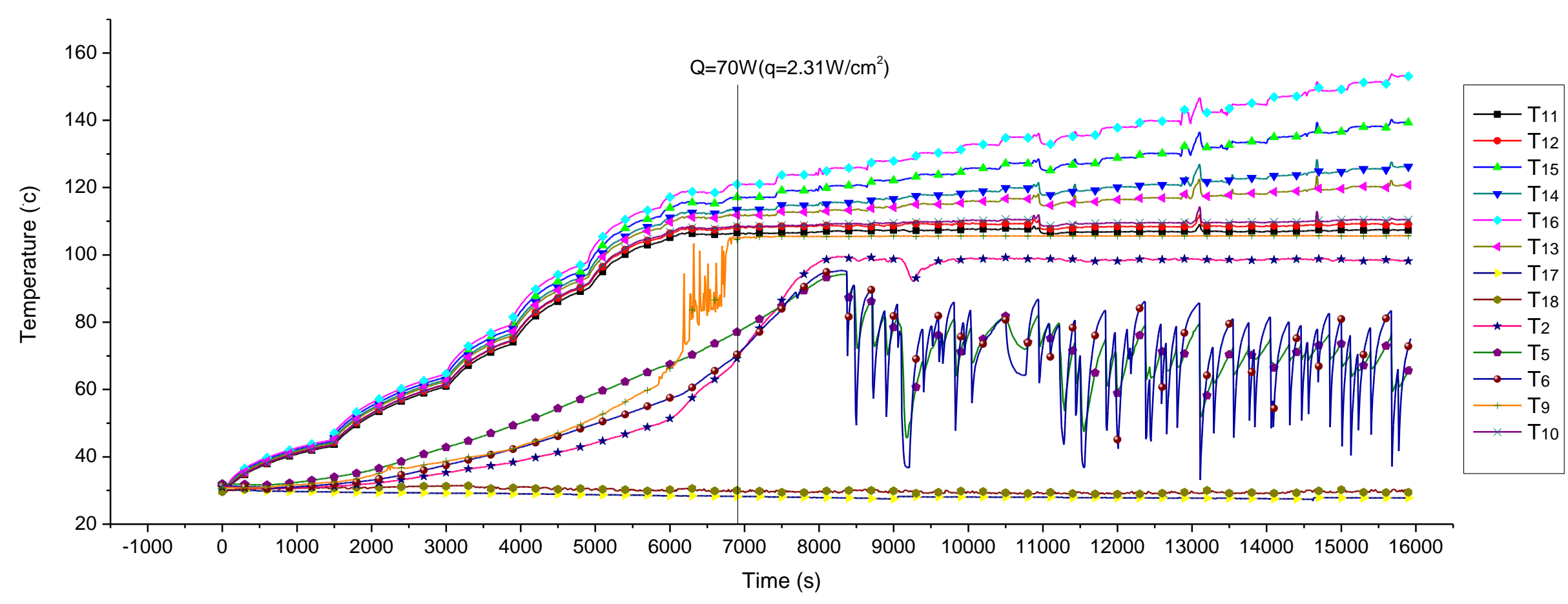


Fig.10 Variable heat load operation process of the 5-mm EC condition




Fig.11 Evaporator thermal resistance of $1 \mathrm{~mm}$

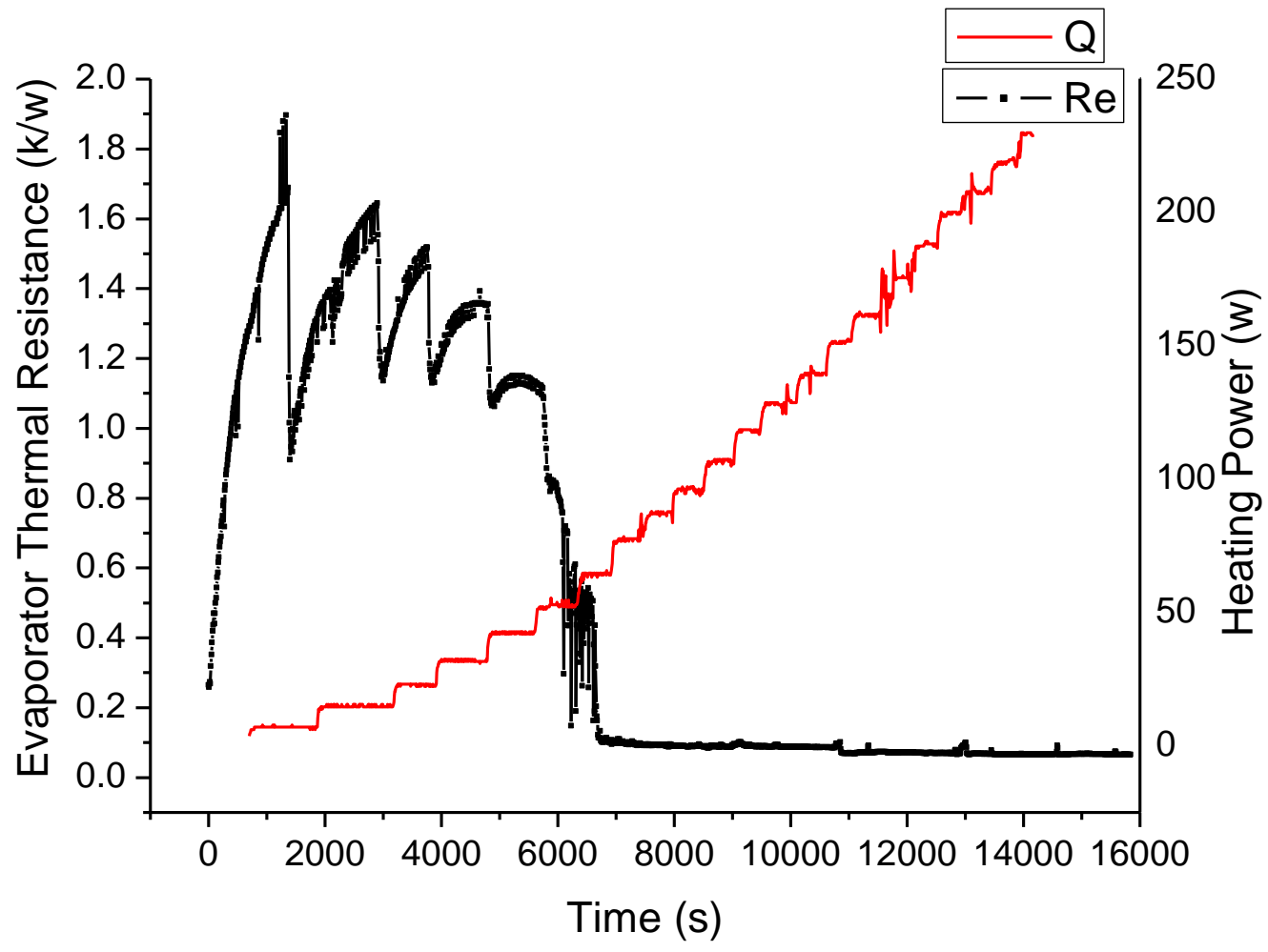


Fig.12 Evaporator thermal resistance of $5 \mathrm{~mm}$

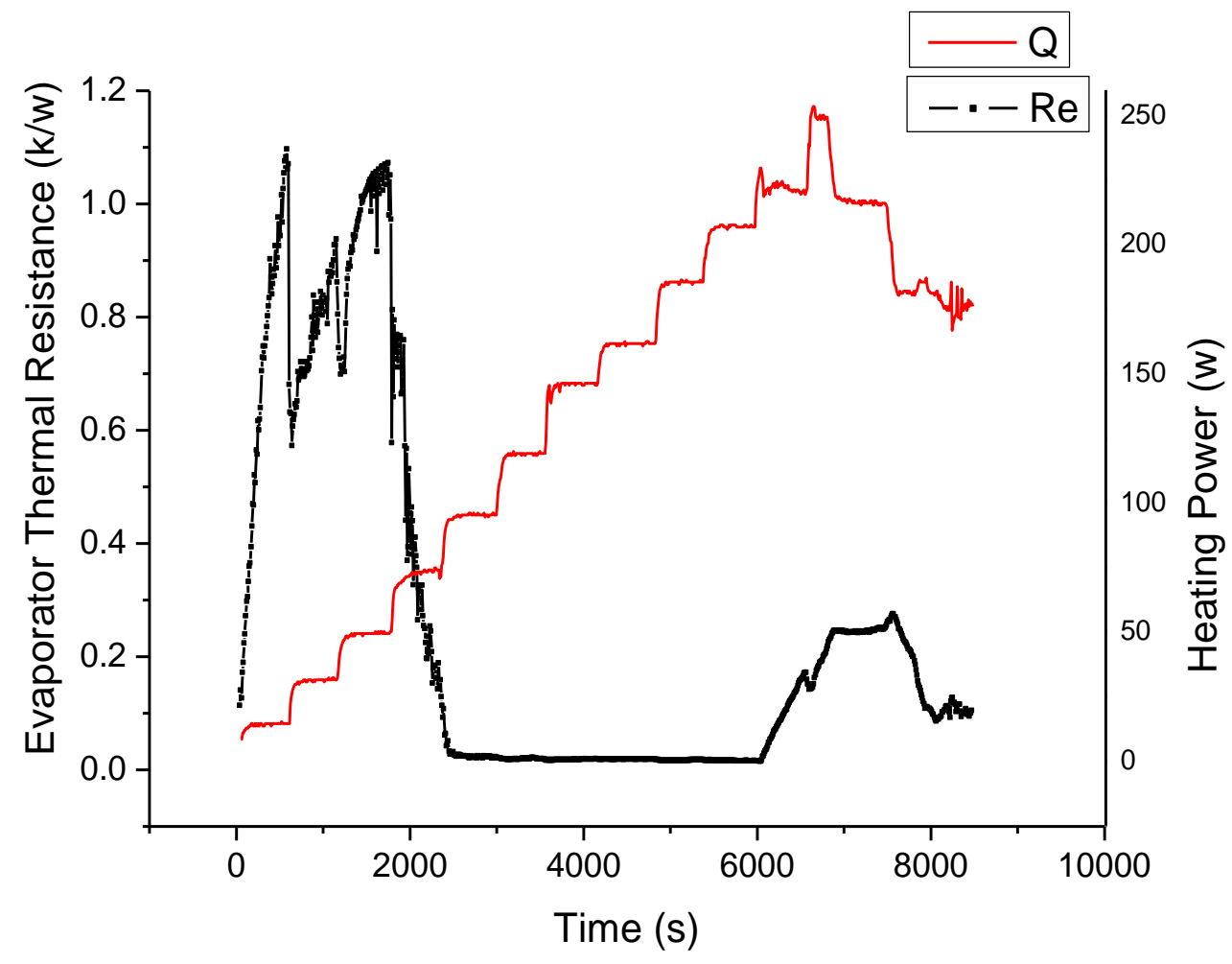


Fig.13 Total thermal resistance of $1 \mathrm{~mm}$

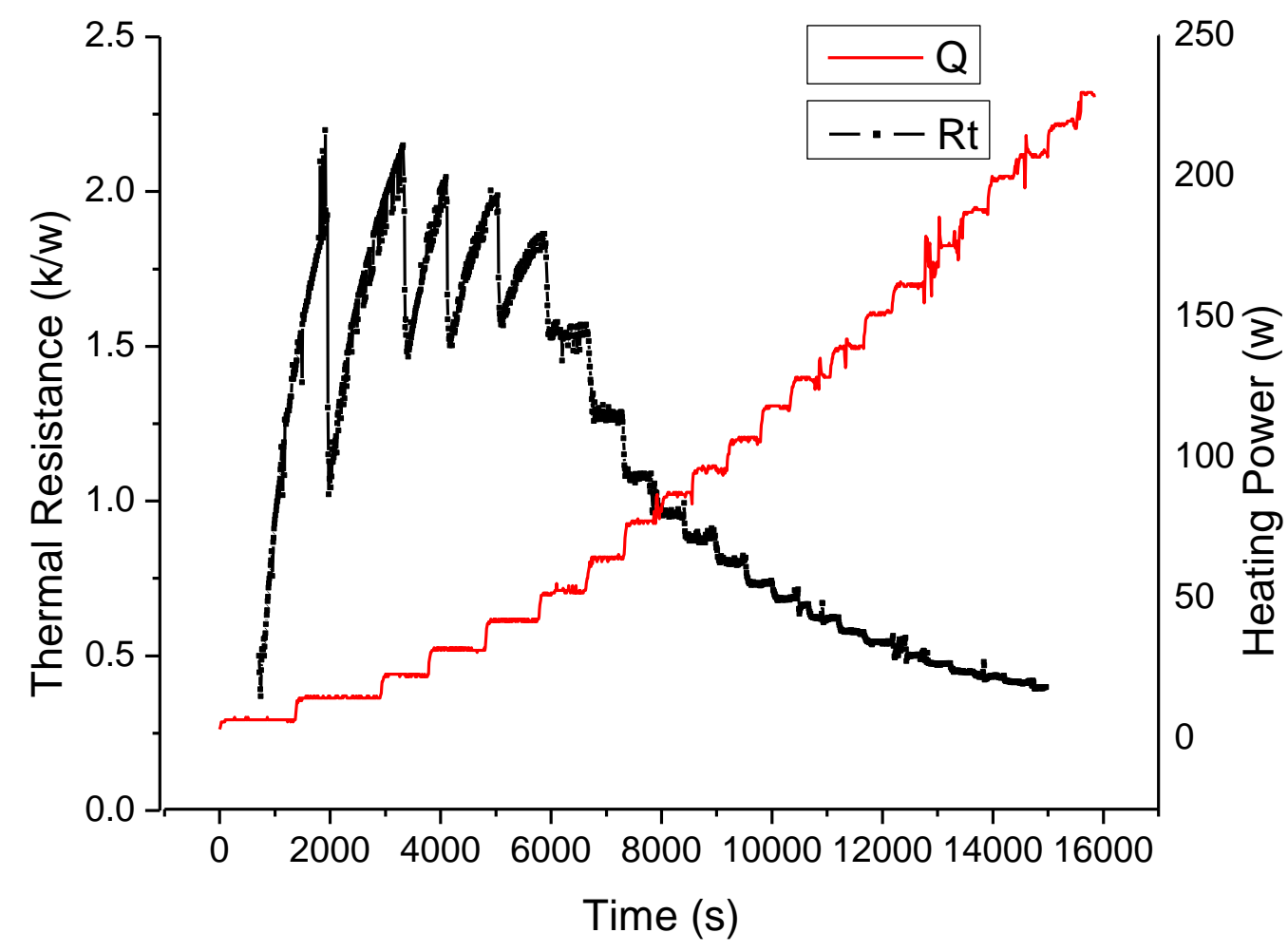


Fig.14 Total thermal resistance of $5 \mathrm{~mm}$

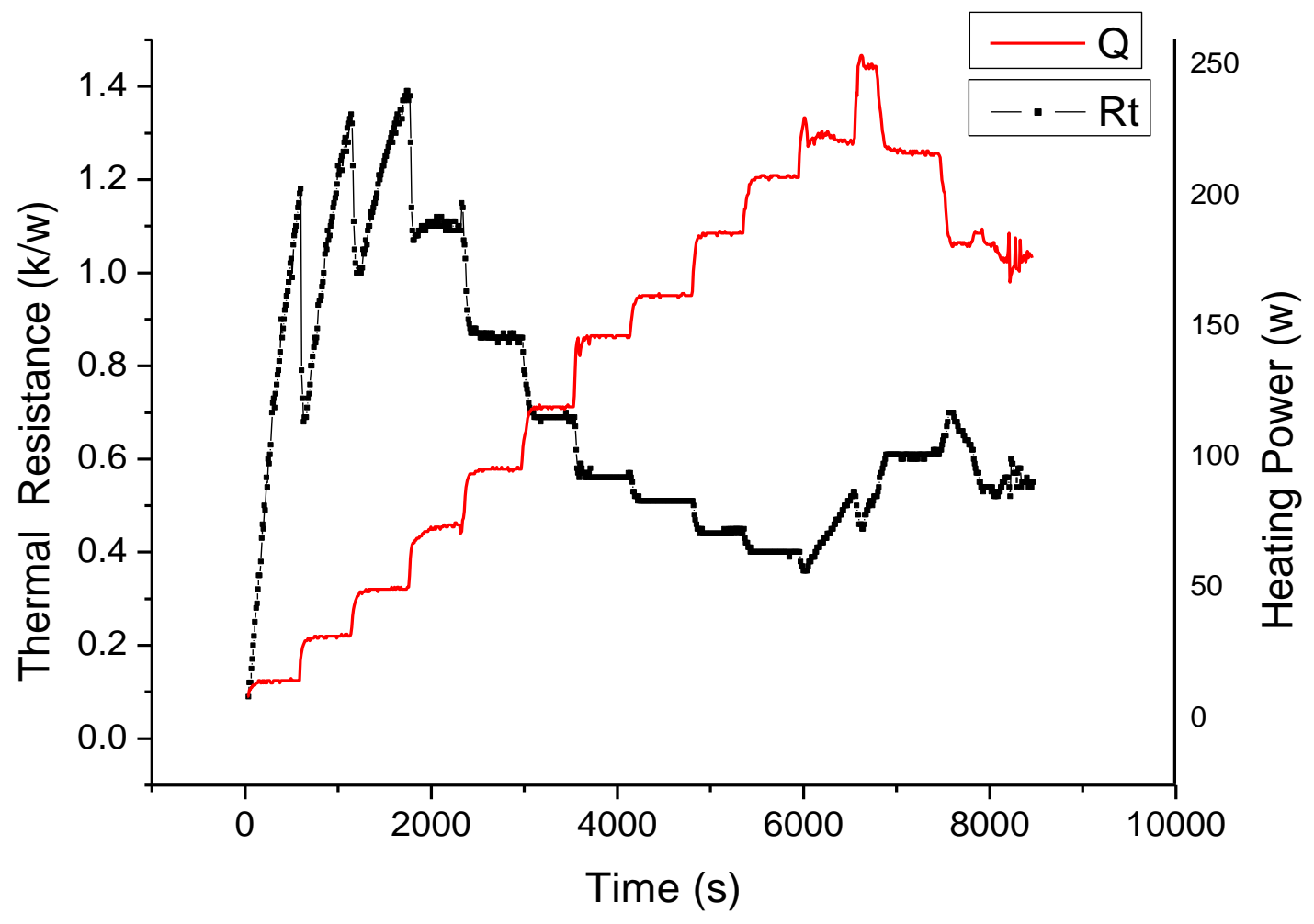


Fig.15 Total thermal resistance of $1 \mathrm{~mm}$

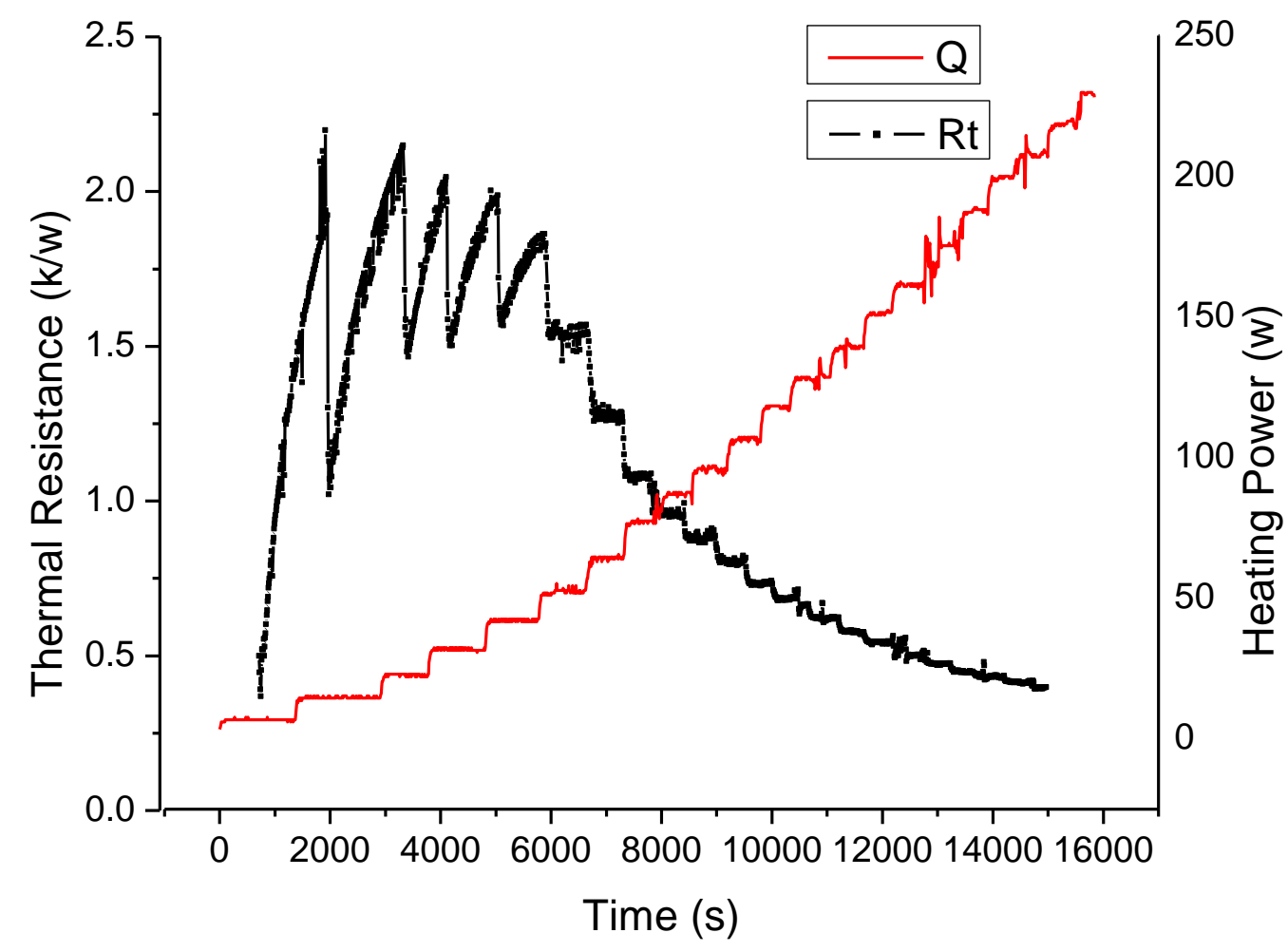


Fig.16 Total thermal resistance of $5 \mathrm{~mm}$



